\title{
Schwinger Functions in Noncommutative Quantum Field Theory
}

\author{
Dorothea Bahns
}

\begin{abstract}
It is shown that the $n$-point functions of scalar massive free fields on the noncommutative Minkowski space are distributions which are boundary values of analytic functions. Contrary to what one might expect, this construction does not provide a connection to the popular traditional Euclidean approach to noncommutative field theory (unless the time variable is assumed to commute). Instead, one finds Schwinger functions with twistings involving only momenta that are on the mass-shell. This explains why renormalization in the traditional Euclidean noncommutative framework crudely differs from renormalization in the Minkowskian regime.
\end{abstract}

\section{Introduction}

A quantum field theoretic model is to a large part determined by the choice of a partial differential operator. For physical reasons, this operator has to be hyperbolic, and one of its fundamental solutions, the so-called Feynman propagator, is the building block in any perturbative calculation of physically relevant quantities. Nonetheless, ever since proposed by Symanzik in 1966 [10] based on ideas of Schwinger, the so-called Euclidean framework has played a very important role. In this framework, the building block is the so-called Schwinger function, a fundamental solution of an elliptic partial differential operator. The Euclidean formalism not only simplifies calculations, but seems to be indispensable in constructive quantum field theory. The remarkable theorem of Osterwalder and Schrader gives sufficient conditions for the possibility to recover the original hyperbolic (physically meaningful) field theory from a Euclidean framework, and therefore justifies the Euclidean framework in ordinary quantum field theory. It is recalled below how the Schwinger function of the Euclidean framework of free scalar field theory is derived by analytic 
continuation from the hyperbolic theory and how it relates to the Feynman propagator.

To incorporate gravitational aspects into quantum field theory, one possibility is to study quantum fields on noncommutative spaces, the most popular of which is the noncommutative "Moyal space" whose coordinates are subject to commutation relations of the Heisenberg type [4]. Already in that early paper, a possible setting for (unitary) hyperbolic perturbative quantum field theory was proposed, where the field algebra is endowed with a noncommutative product, the twisted (convolution) product. Notwithstanding, the vast majority of publications on field theory on noncommutative spaces ("noncommutative field theory") has been and still is formulated within a Euclidean setting. This setting was not derived from a hyperbolic noncommutative theory but directly from the Euclidean framework of ordinary field theory by replacing all products with twisted ones. I shall refer to this approach as the traditional noncommutative Euclidean framework. Despite some attempts, it has not been possible to relate this traditional noncommutative Euclidean setting to some hyperbolic noncommutative theory - in fact, there is evidence that it might be impossible to do so, unless the time variable commutes with all space variables. It became clear after some years that within the traditional Euclidean noncommutative framework, already the models built from the most harmless of fields, namely the scalar massive fields, have very peculiar properties. Most notably, the so-called ultraviolet-infrared mixing problem noted in [8] severely limits the type of model that can be defined at all $[6,7]$.

It is at present not clear whether the ultraviolet-infrared mixing problem is present in a hyperbolic setting, as the calculations and the combinatorial aspects of hyperbolic noncommutative field theory are quite involved. It is therefore desirable to find a Euclidean framework that can actually be derived from a hyperbolic noncommutative setting in the hope that - as in ordinary quantum field theory - such a Euclidean setting might simplify the combinatorial aspects of perturbation theory and that the full Euclidean machinery of renormalization might be employed. In such a setting, it might be feasible to investigate a theory's renormalizability and the ultraviolet-infrared mixing problem in general.

As a very first step towards this goal, I will show in this note that one can indeed derive a noncommutative Euclidean framework from a hyperbolic theory of free fields on the Moyal space, and that this framework is not the traditional one that is investigated in the literature. In contrast to this traditional framework, the new Euclidean framework can moreover be related to a setting involving Feynman propagators via an analytic continuation similar to the one of ordinary quantum field theory.

The main idea is to take the Wightman functions (of free fields) as the starting point. This is justified by the fact that within axiomatic quantum field theory, the Wightman functions are the fundamental objects from which the field content can be recovered by the celebrated reconstruction theorem. On Minkowski space, a simple construction allows to pass from the Wightman functions (of free fields) to the Schwinger functions (of free fields), and 
from there to expressions involving (hyperbolic) Feynman propagators. This procedure will be mimicked in the present note.

The note is organized as follows: In the next section it is recalled how the Schwinger function is derived in ordinary massive scalar quantum field theory and how it is related to the Feynman propagator. In the third section, a Euclidean 4-point function (Schwinger function) is derived from a noncommutative hyperbolic Wightman function of 4 free massive scalar fields and the prescription how Schwinger functions of arbitrarily high order are calculated is given. It is shown that the Euclidean framework thus derived differs from the traditional noncommutative Euclidean approach. Moreover, the relation to Feynman propagators is clarified. In an outlook I will briefly comment on further possible research that ensues from these new results.

\section{Euclidean Methods in Quantum Field Theory}

The hyperbolic partial differential operator of massive scalar field theory is the massive Klein-Gordon operator $P:=\frac{\partial^{2}}{\partial x_{0}{ }^{2}}-\Delta_{\mathbf{x}}+m^{2}$ on $\mathbb{R}^{4}$ where $\Delta_{\mathbf{x}}$ denotes the Laplace operator on $\mathbb{R}^{3}, \mathbf{x} \in \mathbb{R}^{3}$, and $m>0$ is a real parameter, called the field's mass. As mentioned in the introduction, all the relevant quantities of a scalar field theoretic model can be calculated from a fundamental solution of this operator. Recall here that a distribution $E \in \mathcal{D}^{\prime}\left(\mathbb{R}^{n}\right)$ is a fundamental solution (or Green's function) of a partial linear differential operator $P(\partial)$ on $\mathbb{R}^{n}$ provided that in the sense of distributions, $P(\partial) E=-\delta$ with $\delta$ denoting the $\delta$-distribution.

Our starting point here, however, is the 2-point-function $\Delta_{+} \in \mathcal{S}^{\prime}\left(\mathbb{R}^{4}\right)$, a tempered distribution which is a solution (not a fundamental solution) of the Klein-Gordon equation, $P \Delta_{+}=0$ in the sense of distributions. For $x=$ $\left(x_{0}, \mathbf{x}\right) \in \mathbb{R}^{4}, x_{0} \in \mathbb{R}, \mathbf{x} \in \mathbb{R}^{3}$, it is given explicitly by

$$
\Delta_{+}(x)=\frac{1}{(2 \pi)^{3}} \int \frac{1}{2 \omega_{\mathbf{k}}} \mathrm{e}^{-\mathrm{i} \omega_{\mathbf{k}} x_{0}+\mathrm{ikx}} \mathrm{d}^{3} \mathbf{k}, \quad \text { where } \omega_{\mathbf{k}}=\sqrt{\mathbf{k}^{2}+m^{2}},
$$

an expression which in fact makes sense as an oscillatory integral, see [9, Sect. IX.10] for details. Here and in what follows, boldface letters denote elements of $\mathbb{R}^{3}$ and an expression such as $\mathbf{k x}$ is shorthand for the canonical scalar product of $\mathbf{k}$ and $\mathbf{x}$.

It is well-known that $\Delta_{+}$is the boundary value (in the sense of distributions) of an analytic function. To see this, let us first fix some notation. Let $a \in \mathbb{R}^{n}$ with $|a|=1$, let $\theta \in(0, \pi / 2)$, and let $a y$ denote the canonical scalar product in $\mathbb{R}^{n}$. Then the cone about $a$ with opening angle $\theta$ is the set $\Gamma_{a, \theta}=\left\{y \in \mathbb{R}^{n}|y a>| y \mid \cos \theta\right\} \subset \mathbb{R}^{n}$. Let $\Gamma_{a, \theta}^{*}$ denote the dual cone, $\Gamma_{a, \theta}^{*}:=\Gamma_{a, \frac{\pi}{2}-\theta}$. For tempered distributions whose support is contained in the closure of a cone, the following general assertion holds:

Theorem 1 ([9, Thm. IX.16]). Let $u$ be a tempered distribution with support in the closure of a cone $\Gamma_{a, \theta}, a \in \mathbb{R}^{n}, 0<\theta<\pi / 2$. Then its Fourier transform $\tilde{u}$ 
is the boundary value (in the sense of tempered distributions) of a function $f$ which is analytic in the tube $\left\{z \in \mathbb{C}^{n} \mid-\mathrm{im} z \in \Gamma_{a, \theta}^{*}\right\}=: \mathbb{R}^{n}-\mathrm{i} \Gamma_{a, \theta}^{*} \subset \mathbb{C}^{n}$.

Observe that for $\tilde{u}$ to be the boundary value of $f$ as above in the sense of tempered distributions means that for any $\eta \in \Gamma_{a, \theta}^{*}$ and for any test function $g \in \mathcal{S}\left(\mathbb{R}^{4}\right)$, we have for $t \in \mathbb{R}$ approaching 0 from above,

$$
\int f(x-\mathrm{i} t \eta) g(x) \mathrm{d} x \rightarrow \tilde{u}(g)
$$

as tempered distributions.

The Fourier transform $\tilde{\Delta}_{+}$of the 2-point function,

$$
\tilde{\Delta}_{+}\left(p_{0}, \mathbf{p}\right)=\frac{1}{2 \omega_{\mathbf{p}}} \delta\left(p_{0}-\omega_{\mathbf{p}}\right)
$$

is a tempered distribution whose support (the positive mass shell) is contained in the closure of the cone $\Gamma_{+}:=\Gamma_{(1,0), \pi / 4}$ (the forward light cone). Applied to $u:=\tilde{\Delta}_{+}$, Theorem 1 thus guarantees that $\tilde{u}=\Delta_{+}$is the boundary value of a function $f$ which is analytic in $\mathbb{R}^{4}-\mathrm{i} \Gamma_{+}$(observe that $\Gamma_{+}^{*}=\Gamma_{+}$). Explicitly, for $x=\left(x_{0}, \mathbf{x}\right) \in \mathbb{R}^{4}$ and $\eta=\left(x_{4}, \mathbf{0}\right) \in \Gamma_{+}$(hence $x_{4}>0$ ), we have in this case

$$
f(x-\mathrm{i} \eta)=\frac{1}{(2 \pi)^{3}} \int \frac{1}{2 \omega_{\mathbf{k}}} \mathrm{e}^{+\mathrm{i} \mathbf{k} \mathbf{x}-\omega_{\mathbf{k}}\left(x_{4}+\mathrm{i} x_{0}\right)} \mathrm{d}^{3} \mathbf{k} .
$$

We now define a function $s$ via

$$
s\left(\mathbf{x}, x_{4}+\mathrm{i} x_{0}\right):=f(x-\mathrm{i} \eta)
$$

for $x=\left(x_{0}, \mathbf{x}\right) \in \mathbb{R}^{4}$ and $\eta=\left(x_{4}, \mathbf{0}\right) \in \Gamma_{+}$as above. Making use of the identity

$$
\frac{1}{2 \omega_{\mathbf{k}}} \mathrm{e}^{-\omega_{\mathbf{k}} x_{4}}=\frac{1}{2 \pi} \int_{-\infty}^{\infty} \frac{\mathrm{e}^{\mathrm{i} k_{4} x_{4}}}{k^{2}+m^{2}} \mathrm{~d} k_{4} \quad \text { for } x_{4}>0
$$

where $k=\left(\mathbf{k}, k_{4}\right) \in \mathbb{R}^{4}, k^{2}=\mathbf{k}^{2}+k_{4}^{2}$, and setting $x_{0}=0$ in (2.2), we then find that

$$
s(x)=\frac{1}{(2 \pi)^{4}} \int \frac{\mathrm{e}^{\mathrm{i} k x}}{k^{2}+m^{2}} \mathrm{~d}^{4} k \quad \text { where } x=\left(\mathbf{x}, x_{4}\right) \in \mathbb{R}^{4}, x_{4}>0 .
$$

One now extends the function $s$ to a distribution $S \in \mathcal{S}^{\prime}\left(\mathbb{R}^{4}\right)$, the so-called Schwinger function, by dropping the restriction on $x_{4}$. Then the integral still makes sense as an oscillatory integral

$$
\frac{1}{(2 \pi)^{4}} \int \frac{\mathrm{e}^{\mathrm{i} k x}}{k^{2}+m^{2}} \mathrm{~d}^{4} k
$$

Observe that $S$ is the unique fundamental solution of the elliptic partial differential operator $\Delta-m^{2}$ with $\Delta$ the Laplace operator on $\mathbb{R}^{4}$.

As mentioned in the introduction, the building block in hyperbolic perturbation theory is the Feynman propagator $\Delta_{F}$, a fundamental solution for the Klein-Gordon operator $P=\frac{\partial^{2}}{\partial x_{0}{ }^{2}}-\Delta_{\mathbf{x}}+m^{2}$. Without going into details, let me mention that, remarkably, the Fourier transform $\tilde{S}$ of the Schwinger function is the analytic continuation of the Fourier transform $\tilde{\Delta}_{F}$ of the 
Feynman propagator $\Delta_{F}$ (up to a sign). In fact, formally, for the kernel $w$ given by

$$
w\left(\mathbf{p}, p_{4}-\mathrm{i} p_{0}\right):=\tilde{\Delta}_{F}\left(p_{0}+\mathrm{i} p_{4}, \mathbf{p}\right)
$$

we have

$$
\tilde{S}\left(\mathbf{p}, p_{4}\right)=-w\left(\mathbf{p}, p_{4}\right)
$$

for the Schwinger function's Fourier transform $\tilde{S}$.

\section{Analytic Continuation in the Noncommutative Case}

It would be beyond the scope of this note to explain the possible unitary perturbative setups for massive scalar fields on the noncommutative Moyal space with hyperbolic signature (see [3] for a comparison). Only two features of such noncommutative (hyperbolic) field theories matter here. The first is the fact that our starting point still is the Klein-Gordon operator and the 2-point-function $\Delta_{+}$discussed in the previous section. The second important feature - and this feature is shared by the traditional noncommutative Euclidean formalism - is the fact that one has to consider not only products but also twisted products of distributions.

To fix the notation, we note here that for two Schwartz functions $f, g \in$ $\mathcal{S}\left(\mathbb{R}^{4}\right)$ this twisted product (Moyal product) is

$$
f * g(x)=\int \tilde{f}(k) \tilde{g}(p) \mathrm{e}^{-\mathrm{i}(p+k) x} \mathrm{e}^{-\frac{i}{2} p \theta k} \mathrm{~d}^{4} k \mathrm{~d}^{4} p
$$

where $\tilde{f}$ and $\tilde{g}$ denote the Fourier transforms of $f$ and $g$, respectively, and where $\theta$ is a nondegenerate antisymmetric $4 \times 4$-matrix. Observe that in a Euclidean theory, a product such as $k x$ stands for the canonical scalar product on $\mathbb{R}^{4}$, whereas in a hyperbolic setting, it denotes a Lorentz product, $k x=k_{0} x_{0}-\mathbf{k x}$ for $x=\left(x_{0}, \mathbf{x}\right)$ and $k=\left(k_{0}, \mathbf{k}\right)$, with $\mathbf{k x}$ denoting the canonical scalar product on $\mathbb{R}^{3}$. The oscillating factor $\mathrm{e}^{-\frac{\mathrm{i}}{2} p \theta k}$ is also called the twisting.

\subsection{The Tensor Product of 2-Point Functions}

Since the 2-point function remains unchanged in noncommutative field theory, we have to consider higher order correlation functions in order to see a difference between field theory on Moyal space and ordinary field theory. Again, it would be beyond the scope of this note to explain the whole setup. It will be sufficient to consider as an example a particular contribution to the so-called 4-point function of free massive scalar field theory. In ordinary field theory, the distribution of interest here is the 2-fold tensor product of 2-point functions,

$$
\Delta_{+}^{(2)}(x, y)=\frac{1}{(2 \pi)^{6}} \int \frac{1}{2 \omega_{\mathbf{k}}} \frac{1}{2 \omega_{\mathbf{p}}} \mathrm{e}^{-\mathrm{i}\left(\omega_{\mathbf{k}} x_{0}+\omega_{\mathbf{p}} y_{0}\right)+\mathrm{i}(\mathbf{k} \mathbf{x}+\mathbf{p y})} \mathrm{d}^{3} \mathbf{k} \mathrm{d}^{3} \mathbf{p}
$$

The 4-point function (the vacuum expectation value of four fields) is given as a linear combination of such tensor products, i.e.

$$
\left\langle\Omega, \phi\left(x_{1}\right) \phi\left(x_{2}\right) \phi\left(x_{3}\right) \phi\left(x_{4}\right) \Omega\right\rangle=\sum \Delta_{+}^{(2)}\left(x_{i_{1}}-x_{j_{1}}, x_{i_{2}}-x_{j_{2}}\right)
$$


where the sum runs over all pairs $\left(i_{1}, j_{1}\right),\left(i_{2}, j_{2}\right)$ of indices with $\left\{i_{1}, i_{2}, j_{1}, j_{2}\right\}=$ $\{1,2,3,4\}$ and $i_{1}<j_{1}, i_{2}<j_{2}$. Observe that each of these index sets labels one of the three possibilities to contract the four fields, i.e. $\left(i_{1}, j_{1}\right)=(1,3)$, $\left(i_{2}, j_{2}\right)=(2,4)$ corresponds to the contribution to the 4-point function where the first field is contracted with the third, and the second with the fourth.

By standard arguments from microlocal analysis involving the wavefront set of distributions, it can be shown that even the pullback of this tensor product with respect to the diagonal map, that is, the product in the sense of Hörmander, is a well-defined distribution $\in \mathcal{D}^{\prime}\left(\mathbb{R}^{4}\right)$ (see for instance [9, Chap. IX.10]) which can then be shown to be still tempered. For the kernel given by (3.2), this would amount to setting $x=y$. In order to avoid issues regarding renormalization later, in this note, however, only tensor products of distributions will be considered.

It is well-known and, by application of Theorem 1 , in fact not difficult to see that $\Delta_{+}^{(2)}$ is again the boundary value of an analytic function:

Lemma 2. The tempered distribution $\Delta_{+}^{(2)}$ is the boundary value of a function $f_{2}$ which is analytic in $\mathbb{R}^{4} \times \mathbb{R}^{4}-\mathrm{i} \Gamma_{+} \times \Gamma_{+}$. Explicitly, for

$$
z=\left(x_{0}, \mathbf{x}, y_{0}, \mathbf{y}\right) \in \mathbb{R}^{4} \times \mathbb{R}^{4} \quad \text { and } \quad \eta=\left(x_{4}, \mathbf{0}, y_{4}, \mathbf{0}\right) \in \Gamma_{+} \times \Gamma_{+}
$$

(hence $x_{4}$ and $y_{4}>0$ ), we have

$$
f_{2}(z-\mathrm{i} \eta)=\frac{1}{(2 \pi)^{6}} \int \frac{1}{2 \omega_{\mathbf{k}}} \frac{1}{2 \omega_{\mathbf{p}}} \mathrm{e}^{-\omega_{\mathbf{k}}\left(x_{4}+\mathrm{i} x_{0}\right)-\omega_{\mathbf{p}}\left(y_{4}+\mathrm{i} y_{0}\right)+\mathrm{i} \mathbf{k} \mathbf{x}+\mathrm{i} \mathbf{p y}} \mathrm{d}^{3} \mathbf{k} \mathrm{d}^{3} \mathbf{p} .
$$

For the function $s_{2}$ defined for $\eta$ and $z$ as above, by

$$
s_{2}\left(\mathbf{x}, x_{4}+\mathrm{i} x_{0}, \mathbf{y}, y_{4}+\mathrm{i} y_{0}\right):=f_{2}(z-\mathrm{i} \eta),
$$

we find for $(x, y)=\left(\mathbf{x}, x_{4}, \mathbf{y}, y_{4}\right) \in \mathbb{R}^{4} \times \mathbb{R}^{4}, x_{4}$ and $y_{4}>0$, the explicit form

$$
s_{2}(x, y)=\frac{1}{(2 \pi)^{8}} \int \frac{1}{k^{2}+m^{2}} \frac{1}{p^{2}+m^{2}} \mathrm{e}^{+\mathrm{i} k x+\mathrm{i} p y} \mathrm{~d}^{4} k \mathrm{~d}^{4} p
$$

where $p^{2}=\mathbf{p}^{2}+p_{4}^{2}$, and likewise, $k^{2}=\mathbf{k}^{2}+k_{4}^{2}$.

Proof. The first claim is a direct consequence of Theorem 1 applied with respect to $x$ and $y$ separately, and the second claim follows again from the identity (2.3).

As in the previous section, one again drops the restrictions on $x_{4}$ and $y_{4}$ and thereby extends $s_{2}$ to a distribution $S_{2}$, whose Fourier transform is the smooth function

$$
\tilde{S}(k) \tilde{S}(p)=\frac{1}{k^{2}+m^{2}} \frac{1}{p^{2}+m^{2}}
$$

Again, upon restriction of $S_{2}$ to $\mathbb{R}^{3} \times \mathbb{R}_{>0} \times \mathbb{R}^{3} \times \mathbb{R}_{>0}$, it is equal to the function $s_{2}$. As an aside, it is mentioned that when one considers the pullback of $\Delta_{+}^{(2)}$ with respect to the diagonal map (such that, formally, one finds $x=y$ in (3.3)), then the kernel $S_{2}(x, x)$ is the Fourier transform of the convolution

$$
\tilde{S} \times \tilde{S}(k)=\int \frac{1}{(k-p)^{2}+m^{2}} \frac{1}{p^{2}+m^{2}} \mathrm{~d}^{4} p .
$$


Moreover, let us consider again, how Feynman propagators enter the game. As is well-known, $\tilde{S}_{2}$ is the analytic continuation of a product of Feynman propagators. Explicitly, we find that its Fourier transform $\tilde{S}_{2}$ is given in terms of the kernel

$$
w_{2}\left(\mathbf{k}, k_{4}-\mathrm{i} k_{0}, \mathbf{p}, p_{4}-\mathrm{i} p_{0}\right):=\tilde{\Delta}_{F}\left(k_{0}+\mathrm{i} k_{4}, \mathbf{k}\right) \tilde{\Delta}_{F}\left(p_{0}+\mathrm{i} p_{4}, \mathbf{p}\right)
$$

as follows

$$
\tilde{S}_{2}\left(\mathbf{k}, k_{4}, \mathbf{p}, p_{4}\right)=-w_{2}\left(\mathbf{k}, k_{4}, \mathbf{p}, p_{4}\right) .
$$

It is well-known that the procedure applied to the twofold tensor product in lemma 2 can be applied more generally. Each contribution to the (hyperbolic) $2 n$-point function (or Wightman function) is an $n$-fold tensor product of 2-point functions ( $n$-point functions for odd $n$ vanish). In order to find the corresponding higher order Schwinger function, one considers the analytic continuation according to Theorem 1 in each of the $n$ variables and proceeds in the same manner as explained for the 4-point function above.

\subsection{The Twisted Product of 2-Point Functions}

In $[1,2]$, it was shown how $2 n$-point functions are calculated in hyperbolic massive scalar field theory on the noncommutative Moyal space ( $n$-point functions for $n$ odd still vanish). Their general properties were investigated in [2]. As it turns out, the first deviation from ordinary field theory shows up in the 4-point function, where one of the contributions is a twisted tensor product of two 2-point functions,

$$
\Delta_{+}^{(\star 2)}(x, y):=\int \frac{1}{2 \omega_{\mathbf{k}}} \frac{1}{2 \omega_{\mathbf{p}}} \mathrm{e}^{-\mathrm{i}\left(\omega_{\mathbf{k}} x_{0}+\omega_{\mathbf{p}} y_{0}\right)+\mathrm{i}(\mathbf{k} \mathbf{x}+\mathbf{p y})} \mathrm{e}^{-\mathrm{i} \tilde{p} \theta \tilde{k}} \mathrm{~d}^{3} \mathbf{k d}^{3} \mathbf{p}
$$

where $\tilde{k}=\left(\omega_{\mathbf{k}}, \mathbf{k}\right)$, and $\tilde{p}=\left(\omega_{\mathbf{p}}, \mathbf{p}\right)$. In the terminology of physics, this means that the momenta $k$ and $p$ in the oscillating factor are on-shell. This will turn out to be very important later on. It is also important to note that, while our starting point is the twisted product (3.1) on $\mathbb{R}^{4}$, the vectors in the twisting are on-shell as a consequence of the support properties of

$$
\tilde{\Delta}_{+}\left(k_{0}, \mathbf{k}\right)=\frac{1}{2 \omega_{\mathbf{k}}} \delta\left(k_{0}-\omega_{\mathbf{k}}\right)
$$

Observe also that compared to the ordinary twisting in (3.1), the factor 2 in the oscillating factor appears, since in the calculations, two oscillating factors as in (3.1) either cancel or (in the above case) add up, see [1,2]. In fact, if we consider the three different contributions to the 4-point function as explained after equation (3.2), we find that two of them are the same as in the commutative case (the twistings cancel), and only in the contribution where the first field is contracted with the third, and the second with the fourth, the twistings add up (hence the factor 2).

Once more, we now apply Theorem 1. 
Lemma 3. The tempered distribution $\Delta_{+}^{(\star 2)}$ is the boundary value of a function $f_{2}^{\theta}$ which is analytic in $\mathbb{R}^{4} \times \mathbb{R}^{4}-\mathrm{i} \Gamma_{+} \times \Gamma_{+}$. Explicitly, for $z=\left(x_{0}, \mathbf{x}, y_{0}, \mathbf{y}\right) \in$ $\mathbb{R}^{4} \times \mathbb{R}^{4}$ and $\eta=\left(x_{4}, \mathbf{0}, y_{4}, \mathbf{0}\right) \in \Gamma_{+} \times \Gamma_{+}$(hence $x_{4}$ and $\left.y_{4}>0\right)$, we have

$$
f_{2}^{\theta}(z-\mathrm{i} \eta)=\frac{1}{(2 \pi)^{6}} \int \frac{1}{2 \omega_{\mathbf{k}}} \frac{1}{2 \omega_{\mathbf{p}}} \mathrm{e}^{-\omega_{\mathbf{k}}\left(x_{4}+\mathrm{i} x_{0}\right)-\omega_{\mathbf{p}}\left(y_{4}+\mathrm{i} y_{0}\right)+\mathrm{i} \mathbf{k} \mathbf{x}+\mathrm{i} \mathbf{p y}} \mathrm{e}^{-\mathrm{i} \tilde{p} \theta \tilde{k}} \mathrm{~d}^{3} \mathbf{k} \mathrm{d}^{3} \mathbf{p}
$$

where $\tilde{k}=\left(\omega_{\mathbf{k}}, \mathbf{k}\right), \tilde{p}=\left(\omega_{\mathbf{p}}, \mathbf{p}\right)$. For the function $s_{2}^{\theta}$ defined for $\eta$ and $z$ as above, by

$$
s_{2}^{\theta}\left(\mathbf{x}, x_{4}+\mathrm{i} x_{0}, \mathbf{y}, y_{4}+\mathrm{i} y_{0}\right):=f_{2}^{\theta}(z-\mathrm{i} \eta)
$$

we then find for $(x, y)=\left(\mathbf{x}, x_{4}, \mathbf{y}, y_{4}\right) \in \mathbb{R}^{4} \times \mathbb{R}^{4}$ with $x_{4}, y_{4}>0$,

$$
s_{2}^{\theta}(x, y)=\frac{1}{(2 \pi)^{8}} \int \frac{1}{k^{2}+m^{2}} \frac{1}{p^{2}+m^{2}} \mathrm{e}^{+\mathrm{i} k x} \mathrm{e}^{+\mathrm{i} p y} \mathrm{e}^{-\mathrm{i} \tilde{p} \theta \tilde{k}} \mathrm{~d}^{4} k \mathrm{~d}^{4} p
$$

where $p^{2}=\mathbf{p}^{2}+p_{4}^{2}$ and $p^{2}=\mathbf{p}^{2}+p_{4}^{2}$, and with $\tilde{k}=\left(\omega_{\mathbf{k}}, \mathbf{k}\right), \tilde{p}=\left(\omega_{\mathbf{p}}, \mathbf{p}\right)$ as above.

Proof. To see that $\Delta_{+}^{(\star 2)}$ is a tempered distribution, we first note that the twisting is in the multiplier algebra of $\mathcal{S}\left(\mathbb{R}^{8}\right)$. Hence, for any Schwartz function $f \in \mathcal{S}\left(\mathbb{R}^{8}\right)$, we have

$$
\Delta_{+}^{(* 2)}(f)=\Delta_{+}^{(2)}\left(g_{\theta}\right)
$$

where $g_{\theta} \in \mathcal{S}\left(\mathbb{R}^{8}\right)$ is the (inverse) Fourier transform of the product of $\tilde{f}$ and the twisting. The claim follows, since the tensor product $\Delta_{+}^{(2)}$ is tempered.

Since the support of the Fourier transform of $\Delta_{+}^{(\star 2)}$ is still contained in the closure of $\Gamma_{+} \times \Gamma_{+}$, it follows from Theorem 1 that $\Delta_{+}^{(\star 2)}$ indeed is the boundary value of the analytic function $f_{\theta}$. The explicit form of $s_{\theta}$ again follows from the identity (2.3) — which, as should be noted, does not affect the twisting factor.

Observe that $s_{2}^{\theta}$ and $s_{2}$ from Lemma 2 differ only by the oscillating factor $\mathrm{e}^{-\mathrm{i} \tilde{p} \theta \tilde{k}}$. As before, we now extend $s_{2}^{\theta}$ to a distribution $S_{2}^{\theta}$ by dropping the restriction on $x_{4}$ and $y_{4}$, such that $S_{2}^{\theta}$ is given by the Fourier transform of the smooth function

$$
\tilde{S}_{2}^{\theta}(k, p)=\frac{1}{k^{2}+m^{2}} \frac{1}{p^{2}+m^{2}} \mathrm{e}^{-\mathrm{i} \tilde{p} \theta \tilde{k}} .
$$

Again, in the case of coinciding points, instead of $\tilde{S}_{2}^{\theta}(k, p)$ one considers the Fourier transform of the (now twisted) convolution

$$
\int \frac{1}{(k-p)^{2}+m^{2}} \frac{1}{p^{2}+m^{2}} \mathrm{e}^{-\mathrm{i} \tilde{p} \theta \widetilde{k-p}} \mathrm{~d}^{4} p
$$

where $\widetilde{k-p}=\left(\omega_{\mathbf{k}-\mathbf{p}}, k-p\right)$.

It is very important to note that the momenta which appear in the oscillating factors in all the expressions above are on-shell, i.e. that they are of the form $\tilde{p}=\left(\omega_{\mathbf{p}}, \mathbf{p}\right)$, likewise for $k$ or $p-k$. The oscillating factor therefore distinguishes the components of $\left(\mathbf{p}, p_{4}\right)$ and is, in particular, independent of 
the fourth component $p_{4}$. The reason for this lies in the fact that the Fourier transform of the 2-point function forces the momenta in the oscillating factor to be on-shell, and this is not changed by the analytic continuation.

These considerations turn out to be crucial in the following assertion:

Remark 4. Since the oscillating factor in (3.6) is independent of one of the components of $k$ and $p \in \mathbb{R}^{4}$, it is obvious that $\tilde{S}_{2}^{\theta}$ is the analytic continuation a product of Feynman propagators with an on-shell twisting. Explicitly, we find that the Schwinger function's $S_{2}^{\theta}$ Fourier transform $\tilde{S}_{2}^{\theta}$ is given in terms of the kernel

$$
w_{2}^{\theta}\left(\mathbf{k}, k_{4}-\mathrm{i} k_{0}, \mathbf{p}, p_{4}-\mathrm{i} p_{0}\right):=\tilde{\Delta}_{F}\left(k_{0}+\mathrm{i} k_{4}, \mathbf{k}\right) \tilde{\Delta}_{F}\left(p_{0}+\mathrm{i} p_{4}, \mathbf{p}\right) \mathrm{e}^{-\mathrm{i} \tilde{p} \theta \tilde{k}}
$$

as follows

$$
\tilde{S}_{2}^{\theta}\left(\mathbf{k}, k_{4}, \mathbf{p}, p_{4}\right)=-w_{2}^{\theta}\left(\mathbf{k}, k_{4}, \mathbf{p}, p_{4}\right) .
$$

Remark 5. All this remains true when one calculates the higher order Schwinger functions from the $2 n$-point functions. These latter distributions are of a similar form as (3.4), i.e. they are twisted tensor products of 2-point functions where a certain combinatorics determines which combinations of momenta appear in the twistings, see [2]. The important point is that again, all momenta in the twistings are on-shell. Therefore, the same construction that was employed for the 4-point function above, i.e. an analytic continuation in the $n$ variables separately, can be applied and again leads to Schwinger functions with twistings that remain on-shell. Finally, the analytic continuation of the corresponding Fourier transform can be performed as in Remark 4 and leads to (twisted products of) Feynman propagators with twistings still only involving mass-shell momenta.

The fact that one starts from hyperbolic two-point functions which in turn force the momenta in the twistings to be on-shell is the essential difference to the traditional noncommutative Euclidean framework employed in the literature. In this latter framework, (Euclidean) Schwinger functions are the starting point, and of course, when twisted products appear, by (3.1) the oscillating factors depend on all four components of a momentum vector $k=\left(\mathbf{k}, k_{4}\right)$. For instance, instead of finding $\tilde{s}_{2}^{\theta}$ as in (3.6), one starts from the following expression

$$
\tilde{e}_{2}^{\theta}(k, p)=\frac{1}{k^{2}+m^{2}} \frac{1}{p^{2}+m^{2}} \mathrm{e}^{-\mathrm{i} p \theta k}
$$

where $k=\left(\mathbf{k}, k_{4}\right)$ and $p=\left(\mathbf{p}, p_{4}\right)$. So far, it was not possible to relate this framework to a hyperbolic one, the main difficulty being the dependence of the oscillating factor on $k_{4}$. Naively copying the procedure sketched on the previous pages and in Remark 4 to pass to Feynman propagators (via the kernels $w, w_{2}$ and $w_{2}^{\theta}$, respectively) leads to exponentially increasing terms which render the integrals ill-defined. Not even the apparently easy way out to make the oscillating factor independent of one of the components in an ad hoc way, by requiring $\theta$ to be a matrix of rank 2 ("spacelike noncommutativity"), provides a sensible theory in the long run, cf. [5] and [1, Sect. 5]. 
Remark 4 shows that such measures are unnecessary when the new noncommutative Euclidean framework derived from the hyperbolic $n$-point functions is employed.

\section{Outlook}

Based on the above considerations, the most important question now is whether it is possible to set up a consistent Euclidean noncommutative framework with on-shell momenta in the twisting. An obstruction might be that, as can be easily seen already in the example $S_{2}^{\theta}$ discussed above, the higher order Schwinger functions are not symmetric with respect to reflections in the origin. Also, the new Euclidean on-shell product is not associative. This may jeopardize the possibility to set up a complete consistent perturbative framework using a Schwinger functional.

Still, it is to be hoped that the results presented here open many interesting possibilities for future research. For one thing, one should try to generalize the Osterwader Schrader Theorem in this setting. Also, it would be most interesting to study whether the ultraviolet-infrared mixing problem appears in this setting at all. Certainly,there is reason to hope so, since the most prominent graph (the nonplanar tadpole) that exhibits this problem in the traditional Euclidean noncommutative approach, does not do so, when one simply replaces its twisting by an on-shell twisting.

Last but not least, a thorough understanding of the new Euclidean setup (if feasible) should enable us to learn more about hyperbolic noncommutative models - which in themselves have proved to be quite difficult to treat. If a consistent Euclidean perturbative setup can be developed from the ideas presented here, general proofs of renormalizability of hyperbolic noncommutative field theory should at last be possible.

\section{Acknowledgments}

Supported by the German Research Foundation (Deutsche Forschungsgemeinschaft (DFG)) through the Institutional Strategy of the University of Göttingen

Open Access. This article is distributed under the terms of the Creative Commons Attribution Noncommercial License which permits any noncommercial use, distribution, and reproduction in any medium, provided the original author(s) and source are credited.

\section{References}

[1] Bahns, D., Doplicher, S., Fredenhagen, K., Piacitelli, G.: Field theory on noncommutative spacetimes: quasiplanar wick products. Phys. Rev. D 710, 025022 (2005) 
[2] Bahns, D.: Local counterterms on the noncommutative Minkowski space. In: Rigorous Quantum Field Theory. Progr. Math., vol. 251, pp. 11-26. Birkhäuser, Basel (2007)

[3] Bahns, D.: Perturbative methods on the noncommutative Minkowski space. PhD thesis, Hamburg [DESY-THESIS-2004-004] (2003)

[4] Doplicher, S., Fredenhagen, K., Roberts, J.E.: The quantum structure of spacetime at the Planck scale and quantum fields. Commun. Math. Phys. 172(1), 187-220 (1995)

[5] Gayral, V., Gracia-Bondia, J.M., Ruiz, F.R.: Trouble with space-like noncommutative field theory. Phys. Lett. B 610, 141-146 (2005)

[6] Grosse, H., Wulkenhaar, R.: Renormalisation of $\phi^{4}$-theory on noncommutative $\mathbb{R}^{4}$ in the matrix base. Commun. Math. Phys. 256(2), 305-374 (2005)

[7] Gurau, R., Rivasseau, V., Tanasa, A.: A translation-invariant renormalizable non-commutative scalar model. Commun. Math. Phys. 287, 275-290 (2009)

[8] Minwalla, S., Van Raamsdonk, M., Seiberg, N.: Noncommutative perturbative dynamics. J. High Energy Phys. 2, 20-31 (2000)

[9] Reed, M., Simon, B.: Methods of modern mathematical physics. II. In: Fourier Analysis, Self-Adjointness. Academic Press [Harcourt Brace Jovanovich Publishers], New York (1975)

[10] Symanzik, K.: Euclidean quantum field theory. I. Equations for a scalar model. J. Math. Phys. 7, 510-525 (1966)

Dorothea Bahns

Courant Research Centre "Higher Order Structures in Mathematics"

Universität Göttingen

Bunsenstr. 3-5

37073 Göttingen

Germany

e-mail: bahns@uni-math.gwdg.de

Communicated by Raimar Wulkenhaar.

Received: January 19, 2010.

Accepted: October 6, 2010. 